\title{
Fast and Accurate Near-Field Measurement Method using Sequential Spatial Adaptive Sampling (SSAS) Algorithm
}

\author{
Sébastien Serpaud, Alexandre Boyer, Sonia Ben-Dhia and Fabio Coccetti
}

\begin{abstract}
This paper presents an optimization of the Sequential Spatial Adaptive Sampling (SSAS) algorithm to accelerate nearfield scanning of printed circuit boards or integrated circuits. The first originality of this approach is to configure this algorithm from the spatial distribution characteristic of near field on a planar surface. The second originality is to propose three selection criteria to adjust the targeted accuracy with the objective to reduce the measurement time. The low time-consuming algorithm selects only measurement points, which carry the most information, in order to reduce significantly the number of captured points without increasing the measurement error. The analysis of measurement results, based on this algorithm applied on two case studies, validates its effectiveness compared to the classical full regular grid sampling.
\end{abstract}

Keywords - EMC, near-field scanning, adaptive sampling, FPGA, fast measurement.

\begin{tabular}{|c|c|c|}
\hline Parameter & Definition & Unit \\
\hline$\alpha$ & $\begin{array}{l}\text { Absolute attenuation of field magnitude from the } \\
\text { maximum level. }\end{array}$ & {$[\mathrm{dB}]$} \\
\hline Ax, Ay & $\begin{array}{l}\text { Represents the size in } 2 \mathrm{D} \text { plane along } \mathrm{x} \text { axis and } \mathrm{y} \\
\text { axis of space domain of measurement. }\end{array}$ & {$[\mathrm{mm}, \mathrm{mm}]$} \\
\hline$d$ & Final resolution of the full sampling map & {$[\mathrm{mm}]$} \\
\hline$D$ & $\begin{array}{l}\text { Total number of points of the full sampling map at } \\
\text { a final resolution } d \text {. }\end{array}$ & \\
\hline$F$ & $\begin{array}{l}\text { Represents a regionalized variable representing the } \\
\text { near-field radiated by the DUT. }\end{array}$ & \\
\hline$F\left(p_{i}\right)$ & Represents an achievement of $F$ at the point $p_{i}$. & {$[\mathrm{dBA} / \mathrm{m}]$} \\
\hline$\hat{F}\left(p_{i}\right)$ & Estimation of $F$ value at the position $p_{i}$ & {$[\mathrm{dBA} / \mathrm{m}]$} \\
\hline$F_{M}$ & Final measurement dataset of $F$ & \\
\hline$h_{\text {meas }}$ & Height of the measurement. & {$[\mathrm{mm}]$} \\
\hline$M$ & Total number of points where $F$ is measured. & \\
\hline$N$ & Number of points captured during the first step. & \\
\hline$N q$ & $\begin{array}{l}\text { Number of points captured during the step } \\
q=\{2 . . Q\}\end{array}$ & \\
\hline$\Omega$ & Represents the space domain of measurement. & \\
\hline$p_{q i}$ & $\mathrm{i}^{\text {nd }}$ position evaluated during the step $\mathrm{q}$. & $\{x, y\}$ \\
\hline$P_{c}\left(p_{q i}\right)$ & Local dataset of the $N c$ points neighboring $p_{q i}$. & $\{x, y\}^{N c}$ \\
\hline$P_{M}$ & Final dataset of the $M$ points where $F$ is measured. & $\{x, y\}^{M}$ \\
\hline$P_{M}^{\prime}$ & $\begin{array}{l}\text { Temporary dataset of the } M \text { points where } F \text { is } \\
\text { measured during SSAS algorithm process. }\end{array}$ & $\{x, y\}^{M}$ \\
\hline
\end{tabular}

These results have been obtained in the framework of the IRT Saint Exupery's research projects: ROBUSTESSE and FELINE. We acknowledge the financial and in-kind support (background knowledge and services) from the IRT Saint Exupery's industrial and academic members and the financial support of the French National Research Agency. October 2013.

\begin{tabular}{clc}
$P_{N}$ & $\begin{array}{l}\text { Initial dataset of the } N \text { points where } F \text { is measured } \\
\text { during the first step. }\end{array}$ & $\{x, y\}^{N}$ \\
$Q$ & Total number of steps. & {$[\mathrm{step}]$} \\
$r_{\text {Loop }}$ & $\begin{array}{l}\text { Loop radius of the magnetic near field probe. } \\
W\end{array}$ & {$[\mathrm{~mm}]$} \\
Initial sampling step. & {$[\mathrm{mm}]$} \\
\hline
\end{tabular}

\section{INTRODUCTION}

$\mathrm{W}$ ITH the current industrial economic constraints, it is necessary to evaluate electromagnetic behavior of an electronic device as early as possible in its development process. If simulation tools fail to predict accurately the electromagnetic compatibility (EMC) of a complex equipment, the EMC engineer must have efficient measurement-based investigation tools. The near-field scanning (NFS) approach applied on electronic devices has been developed for several years. The experimental investigation performances allowed by NFS is triggering an increasing interest among the electronic equipment and component manufacturers for this EMC investigation measurement method. Owing to the precision and dexterity of modern automation, the NFS test bench based on a well-controlled robot moving probe, represents a powerful and cost-effective investigation tool. This contactless measurement is carried out above an electronic device under test (DUT) without the need to implement specific design constraints. Moreover, high measurement results reproducibility $(<1 \mathrm{~dB})[1]$ is ensured by the intrinsically weak sensitivity of the experimental read-out from the equipment set-up configuration.

Despite the aforementioned key features offered by the NFS measurements, a limiting factor, hindering a wider industrial deployment of this approach, is the effective scanning time duration. In investigation context, measuring only one field component $\left(\mathrm{H}_{\mathrm{z}}\right)$ can be enough to identify the root cause of the EMC related issue. According to the authors' experience, gathered over more than 10 years of industrial EMC expertise, typical near-field measurement may last between 15 minutes up to 5 hours.

However, a complete characterization of the electric and magnetic fields above a complex DUT may require much

S. Serpaud, F Coccetti are with the IRT Saint-Exupéry institute, Toulouse, France (e-mail: sebastien.serpaud@irt-saintexupery.com, Fabio.coccetti@irtsaintexupery.com)

S. Serpaud, S. Ben-Dhia and A. Boyer are with LAAS, Univ. de Toulouse, INSA, Toulouse, France (e-mail: sonia.bendhia@insa-toulouse.fr, alexandre.boyer@laas.fr) 
longer measurement time (e.g. in device modeling context). Depending from the nature of the DUT (integrated circuit, electronic board or electronic equipment), the size of the measurement area could be rather large $\left(1 \mathrm{~cm}^{2}\right.$ up to $\left.400 \mathrm{~cm}^{2}\right)$. As an example, for a $200 \times 150 \mathrm{~mm}$ aeronautic calculator scanned with $1 \mathrm{~mm}$ sampling step, more than 30000 points are necessary to properly execute the measurement. Let consider two magnetic field components are measured, and 3 seconds readout time for each point is within the selected frequency bandwidth (typically $30 \mathrm{MHz}-200 \mathrm{MHz}$ with $\mathrm{RBW}=9 \mathrm{KHz}$ and $200 \mathrm{MHz}-3 \mathrm{GHz}$ with $\mathrm{RBW}=120 \mathrm{KHz}$ ). The total measurement time reaches more than 25 hours. Three main factors contribute to the total duration of the near-field measurement:

- $T_{\text {meas }}$ : the time for the capture equipment (e.g. spectrum analyzer) to measure the voltage across the near-field probe in the selected frequency range.

- $T_{\text {move }}$ : the time for the robot to move the probe across all points above the DUT.

- $T_{c t r l}$ : the computer processing time to control the test bench (communication with all equipments and to process and save measurement data).

In general, with currently available robots and computers, in the worst case scenario, $T_{m o v e}$ and $T_{c t r l}$ account for less than $10 \%$ of the total measurement time. Communication protocols are now optimized to ensure a rapid data transfer from the capture equipment. Thus, the main contributor left is $T_{\text {meas }}$, that is directly linked to the acquisition speed and the number of captured points. Therefore, the most straightforward strategy to reduce the measurement time consists in decreasing the number of points.

The most effective method to reach this goal is to use a spatial adaptive sampling. If a full planar sampling is considered (for example the full sampling map in Fig. 11), most of the scanned points do not carry significant information about the radiated sources. For decades, several adaptive sampling approaches have been developed [2],[3] on the wave propagation application [4],[5] and for planar surface [6],[7]. The planar sampling is suited for electronic equipment scanning. The method described in [7] provides a significant reduction of the number of samples. Note that the authors present no specific indicators to quantify this observation. The choice of the full meshing resolution, taken as reference, is not justified. The main limitation of this approach is the massive used of the Kriging interpolation model. The computation of model at each iteration take a lot of time. The method described in [8] partially improves this point. A second limitation is the use of a random sampling. This sampling approach significantly increases the total path between all the captured points, thereby increasing the measurement duration.

The orientation retained in this paper is to reduce the number of measurement points by capturing only the points that bring the most significant information. The advantages of the Sequential Spatial Adaptive Sampling (SSAS) proposed here are to be a very low time-consuming algorithm and configurable from the characteristics of near-field spatial distribution on a planar surface. Several indicators are also proposed to clearly quantify the performance of the SSAS and facilitate the comparison with other approaches to reduce nearfield scanning duration. The full meshing resolution is fixed by the spatial distribution characteristic of the near-field map, which defines a reference metric to analyze the reduction of measurement duration.

The first version of this algorithm, introduced in [9], suffers of inaccuracies to estimate the maximum emission level. In this paper, a modified version is presented to provide a faithful representation of the near-field above the DUT. Special attention is paid to improve the accuracy around the maximum and the local maxima emission levels of the near-field map.

After a description of the SSAS algorithm in section II and the interpolator used for this study in section III, the section V discuses about the benefits of using this algorithm based on both case studies described in section IV. All the symbols used to describe the algorithm are summarized in annex I.

\section{Description OF the Spatial AdAPtive SAMPLING ALGORITHM}

Let $\Omega \subset \mathbb{R}^{2}$ the space domain of the measurement defined as a rectangular planar surface above the DUT. The near-field emission above the DUT can be represented by a regionalized variable $F$ captured by the near-field probe at several positions of $\Omega$ defined by the dataset $P_{D}=\left\{p_{i}=\left(x_{i}, y_{i}\right), p_{i} \in \Omega\right\}_{i=1}^{D}$. $F\left(p_{i}\right)$ is the measurement of $F$ at the probe positions $p_{i}$ and $D$ is the number of samples of $F$ in $\Omega$. The minimum distance between each probe position along $\mathrm{x}$ and y axes, noted $d$, is the resolution of the final near-field scan. Let $F_{D}=\left\{F\left(P_{D}\right)\right\}$ the full sampling dataset.

In this paper, only the measurement of the magnitude of the field $(F \in \mathbb{R})$ as described in the standard IEC 61967-3 is considered [10]. The measurement is carried out at only one frequency. The purpose of the SSAS is to provide a reduced set of probe positions without introducing a significant loss of information on $F$. At the end, the near-field map with the final resolution $d$ is reconstructed by interpolation.

The SSAS algorithm is based on the Multi-Level Adaptive approach [11] initially introduced in [9]. The first step consists in collecting an initial dataset of $F$. Let $N$ the initial number of sampling points and $P_{N}=\left\{p_{i}=\left(x_{i}, y_{i}\right), p_{i} \in \Omega\right\}_{i=1}^{N}$ the initial dataset of probe positions. The initial dataset of near-field values captured by the probe is defined by $F_{N}=\left\{F\left(P_{N}\right)\right\}$. The objective is to map $\Omega$ with a limited number of points by an optimal spatial cover without any a priori knowledge about $F$. The initial dataset $F_{N}$ has a serious impact about the optimality of the final solution. If the spatial dispersion between samples is high, it is possible to lose relevant information on $F$. In this condition, the adaptive algorithm may converge to a bad solution. On the contrary, a too small spatial dispersion meshing target could induce a large number of initial samples and therefore a weak reduction of the measurement time.

As seen in Fig. 1, several sampling methods [12] could be used to cover $\Omega$. Two types of sampling method exist. The pseudo-random sampling methods capture samples at random positions on $\Omega$. The advantage is that each point of $\Omega$ has the 
same probability to be captured. This is the most popular approach but it can introduce a significant increase of the number of sampling points in order to minimize the spatial dispersion criterion. This parameter is linked to the standard deviation of $\left(\sigma_{g}\right)$ the distribution of $(W)$ the gap between each point and their nearest neighbors on $\Omega$. A low spatial dispersion meshing criterion value means that $\Omega$ is uniformly meshed $\left(\sigma_{g} \rightarrow 0\right)$. The second approach is the regular sampling. The gap $W$ is constant including a more optimal value of the spatial dispersion criterion $\left(\sigma_{g} \rightarrow 0\right)$. This approach is very easy to implement and not time-consuming. The drawback of this approach is that the sampling dataset may not be statistically representative of $F$. The choice of $W$ value is critical and it must be related to the spatial characteristics of $F$.

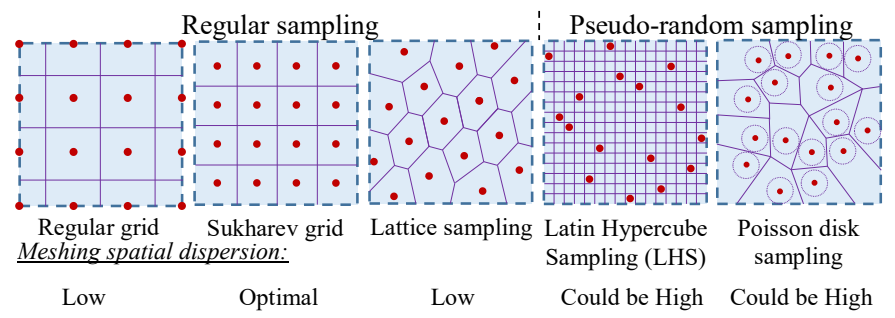

Fig. 1. 16-points Sampling methods (-- boundary of $\Omega$ - p i sampling points)

If the boundaries of $\Omega$ are properly defined, it is reasonable to think that sampling the boundaries of $\Omega$ does not carry a lot of information on $F$. The field values should be low at the boundaries. Adding to a short calculation time, the Sukharev regular sampling is used to collect the initial $F_{N}$ dataset in the proposed SSAS algorithm.

SSAS algorithm is split in a sequential number of step $Q$ as presented in Fig. $2(Q=5$ steps in this example). At the beginning, the full regular grid is built. During the first step1, the initial dataset $F_{N}$ is collected to capture some points from $\Omega$ according to the Sukharev grid with a step $W$. Then, step by step the spatial resolution is progressively decreased according to a selection criterion that defines whether the next point provides enough information about $F$ to be captured.

As shown in Fig. 2, SSAS algorithm progressively meshes $\Omega$. Step by step, the sampling resolution starts with a value of $W$ at the first step and it decreases linearly up to the $d$ value. At each step $q$, a set of point $P_{N q_{\max }}=\left\{p_{q i}, q=2 . . Q\right\}_{i=1}^{N_{\text {max }}}$ is defined to progressively cover $\Omega$, where $N q_{\max }$ is the maximum number of points for the step $q$.

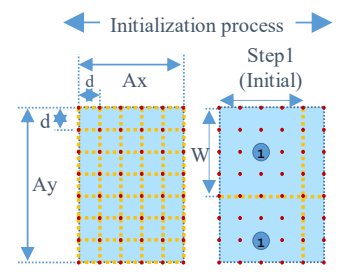

Added pts: $\mathrm{N}_{\text {qmax }}[\min -\max ][$ [ $3 \%-11 \%]$

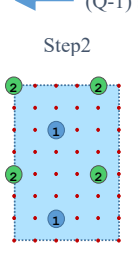

[ 6\%-16\%]

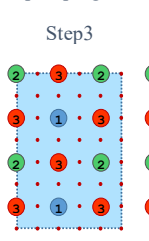

[ 12\%-22\%]
Step4 Step5

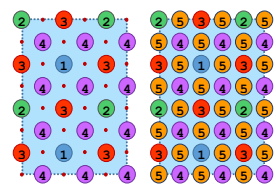

$[\sim 11 \%-25 \%]-[-44 \%-49 \%]$

$\frac{\mathrm{W}}{2 . \sqrt{2}} \quad \frac{\mathrm{W}}{4}=\mathrm{d}$
Fig. 2. Spatial adaptive sampling where $\Omega=\{A x, A y\}$ is progressively covered in 5 steps according to the two input parameters : $d$ and $W$

According to the size of $\Omega=\{\mathrm{Ax}, \mathrm{Ay}\}$ and $W, N q_{\max }$ is bounded by the couple $\left\{N_{q_{\max } \min }, N_{q_{\max } \max }\right\}$. Fig. 3 shows an example of the $N q_{\max }$ boundaries for $W=2 . d=2 \mathrm{~mm}(Q=3$ Steps $)$.

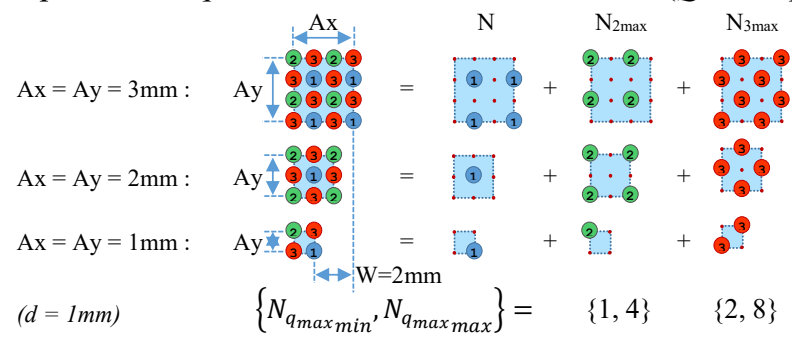

Fig. 3. $\left\{N_{q_{\max } \min }, N_{q_{\max } \max }\right\}$ definition for $\mathrm{W}=2 \mathrm{~mm}$ and $\mathrm{Q}=3$ steps.

A selection criterion ( $\mathrm{cf} \S \mathrm{II} . \mathrm{C}$ ) is used to define whether the point $p_{q i}$ must be captured during the step $q$. Let's define $P_{N q}=$ $\left\{p_{q i}\right\}_{i=1}^{N_{q}}$, as the set of $N_{q} \leq N_{q_{\max }}$ sampling points which are captured (according to the selection criterion) during the step $q$. Step by step, the sum of the captured points $M=N+\sum_{q=2}^{Q} N_{q}$ progressively increases at an exponential rate according to $q$ as shown in Fig. 4.

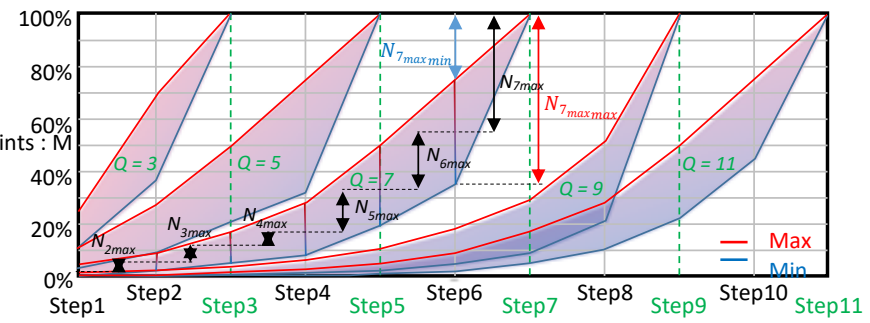

Fig. 4. Evolution of the sampling points $D=M_{\max }=N+\sum_{q=2}^{Q} N_{q_{\max }}$ evaluated by the SSAS algorithm according to the $\mathrm{Q}$ parameter

The dataset $F_{M}=\left\{F\left(P_{M}\right), P_{M}=\left\{p_{i}\right\}_{i=1}^{M} \in \Omega\right\}$ is built from all points captured step by step, where $M$ defines the total number of measured points of $F$ at all specific positions $P_{M}$. The objective is to minimize $M$ (and so $N q$ ) while ensuring that the dataset $F_{M}$ allows building an estimator that minimizes the prediction error of $F$ at any position on $\Omega$.

SSAS algorithm requires only three input parameters: $d, W$ and the selection criterion. In the following, the definition and the justification of these three parameters are discussed.

\section{A. Definition of the final sampling resolutiond}

The final resolution value $d$ can be defined from the type of source. Regardless of the measurement height, the spatial distribution of the radiated emission is linked to the nature of DUT. A power electronic device or a silicon of microcontroller do not produce the same near-field spatial distribution. Consequently, the resolution of near-field probe is selected according to the type of measured sources (DUT). In this case, the final sampling resolution $d$ is linked to the probe resolution. A commonly accepted rule-of-thumb is that a magnetic probe resolution is equal to the radius $r_{\text {loop }}$ of the near-field probe used to measure $F$. Therefore, the lower value of the final sampling resolution $d$ will be set to the probe radius value.

$$
d=r_{\text {loop }}
$$

\section{B. Definition of the initial sampling step $W$}

The initial sampling step $W$ is an important parameter. With 
a large sampling step, relevant information about $F$ may be missed out. A small sampling step increases the number of sampling points, which conflicts with the objective of reducing the measurement time. In this paper, the development of a relationship between the number of points $N$ of the initial dataset and the spatial characteristics of $F$ is proposed. In nearfield region, the distribution of the magnetic field is related to the current that flows through the DUT. That is why the magnetic near-field components are usually measured (using $\mathrm{H}_{\mathrm{xy}}$ or/and $\mathrm{H}_{\mathrm{z}}$ magnetic probes as presented in Fig. 5).

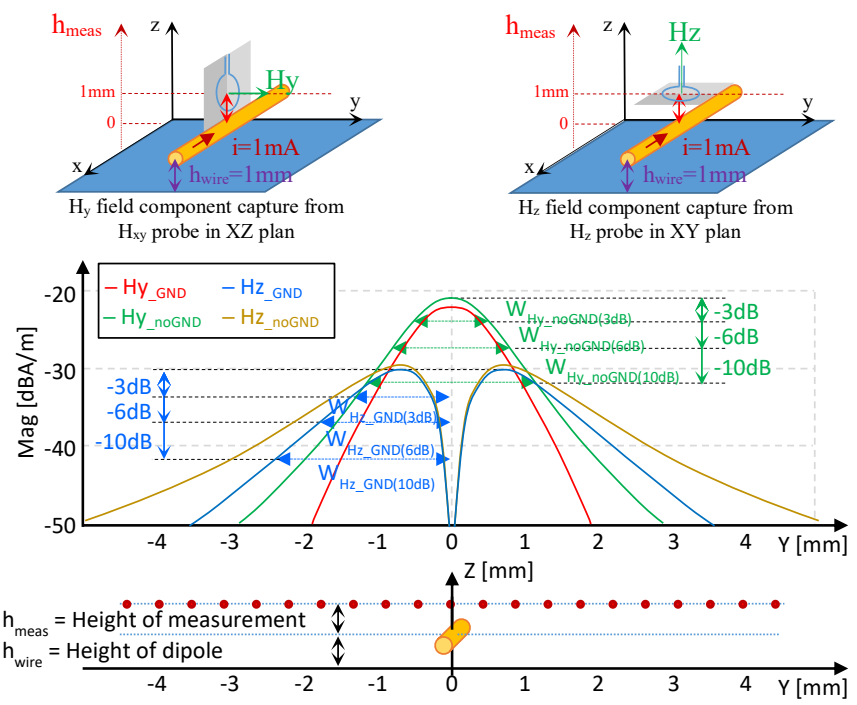

Fig. 5. Spatial distribution of $\mathrm{Hy}(\mathrm{Hx})$ and $\mathrm{Hz}$ magnetic field above a wire $\left(\mathrm{W}_{\text {trace }} \approx 0\right)$ with $1 \mathrm{~mA}$ excitation current at $100 \mathrm{MHz}$, with and without ground plane (at $\mathrm{z}=0$ ) and $h_{\text {wire }}=1 \mathrm{~mm}, h_{\text {meas }}=1 \mathrm{~mm}$

Let's consider a current $i$ flowing through a long thin wire above ground plane. From the spatial distribution of each magnetic near-field component, some relevant spatial characteristics about $F$ can be extracted. For example, the maximum of $\mathrm{H}_{\mathrm{xy}}$ magnetic field appears as a main lobe above the wire. The initial spatial sampling rate $W$ could be defined in order to ensure that a least two measurement points are placed within this lobe. Let $W_{H y}(\alpha)$ and $W_{H z}(\alpha)$ be the width of this lobe according to the attenuation $\alpha(\mathrm{dB})$ compared to the maximum value of magnetic field as defined in Fig. 5 .

Both $W_{H y}(\alpha)$ and $W_{H z}(\alpha)$ parameters can be extracted from derivation of the radiated electric dipole equation [13] summarized in Fig. 6.

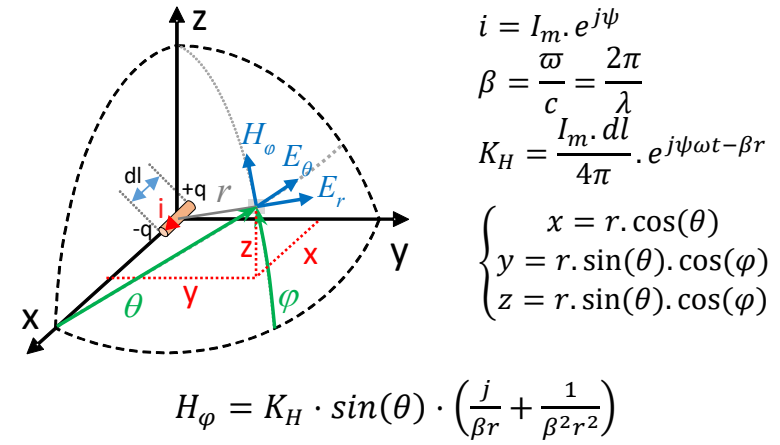

Fig. 6. Radiating electric dipole equation

Let $\theta=\pi / 2$. From the radiated electric dipole equation, the expression of the three magnetic field components can be rewritten in the Cartesian coordinate system according to (3-6):

$$
\begin{aligned}
& H_{x}=0 \\
& H_{y}=K_{H} \cdot \beta^{2} \cdot\left(\frac{j}{\beta r}+\frac{1}{\beta^{2} r^{2}}\right) \cdot \sin (\varphi) \\
& H_{z}=K_{H} \cdot \beta^{2} \cdot\left(\frac{j}{\beta r}+\frac{1}{\beta^{2} r^{2}}\right) \cdot \cos (\varphi) \\
& r=\sqrt{y^{2}+h_{\text {meas }}^{2}}=\frac{y}{\cos (\varphi)}=\frac{h_{\text {meas }}{ }^{2}}{\sin (\varphi)}
\end{aligned}
$$

The modulus of magnetic field components can be written from both geometric parameters $y$ and $h_{\text {meas }}$, as given by (7-8).

$$
\begin{aligned}
& \left|H_{y}\right|=\frac{K_{H} \cdot h_{\text {meas }}}{\left(y^{2}+h_{\text {meas }}{ }^{2}\right)^{3 / 2}} \cdot \sqrt{1+\beta^{2} \cdot\left(y^{2}+h_{\text {meas }}{ }^{2}\right)} \\
& \left|H_{z}\right|=\frac{K_{H} \cdot y}{\left(y^{2}+h_{\text {meas }}{ }^{2}\right)^{3 / 2}} \cdot \sqrt{1+\beta^{2} \cdot\left(y^{2}+h_{\text {meas }}{ }^{2}\right)}
\end{aligned}
$$

The previous equations can be simplified if the term $\beta^{2} .\left(y^{2}+h_{\text {meas }}{ }^{2}\right) \ll 1$. So, in free space, if $r<3 \mathrm{~cm}$ and $f \ll 15 \mathrm{GHz}$, the parameters $W_{H y}(\alpha)$ and $W_{H z}(\alpha)$ are not significantly impacted by the frequency in the near-field region and they only depend on both geometrical parameters $y$ and $h_{\text {meas }}$. Consequently, the maximum absolute value of magnetic field components can be extracted from the derivation of the simplified equations of $H_{y}$ and $H_{z}$, as given by (9-12)

$$
\begin{aligned}
& \frac{\partial\left|H_{y}\right|}{d y} \approx\left(\frac{y}{\left(y^{2}+h_{\text {meas }}\right)^{3 / 2}}\right)^{\prime}=\frac{3 y}{\left(y^{2}+h_{\text {meas }}\right)^{5 / 2}}=0 \\
& \Rightarrow y_{H_{y_{\text {max }}}}=0 \quad \Rightarrow\left|H_{y_{\text {max }} \mid}\right| \approx K_{H} \cdot \frac{1}{h_{\text {meas }}{ }^{2}} \\
& \frac{\partial\left|H_{z}\right|}{d y} \approx\left(\frac{h_{\text {meas }}}{\left(y^{2}+h_{\text {meas }}\right)^{3 / 2}}\right)^{\prime}=0 \\
& \Rightarrow y_{H_{z_{\text {max }}}}= \pm \frac{h_{\text {meas }}}{\sqrt{2}} \Rightarrow\left|H_{z_{\text {max }}}\right| \approx K_{H} \cdot \frac{2}{3 \cdot \sqrt{3} \cdot h_{\text {meas }}{ }^{2}}
\end{aligned}
$$

The distance $y_{0}$ that separates the maximum $H_{y}$ field and the maximum field attenuated by $\alpha$ value is given in (13) and (14). In (15), $W_{H y}(\alpha)$ parameter is obtained by doubling $y o$, as shown in Fig. 5.

$$
\begin{gathered}
\frac{\left|H_{y_{\text {max }}}\right|}{\left|H_{y}\right|} \approx \frac{\left(y^{2}+h_{\text {meas }}\right)^{3 / 2}}{h_{\text {meas }}{ }^{3}}=\alpha \\
y_{0_{H y}}(\alpha)= \pm h_{\text {meas }} \sqrt{\alpha^{2 / 3}-1} \\
W_{H y}(\alpha)=2 \cdot\left|y_{0_{H y}}\right|(\alpha)
\end{gathered}
$$

The same type of development is applied to extract $W_{H Z}(\alpha)$ from $H_{z}$ expression and for the configuration where the dipole is above an ideal ground plane. In the last case, the image theory is used to take into account the ideal ground plane. With the hypothesis that the trace width $\left(W_{\text {trace }}\right)$ is lower than the height of measurement $h_{\text {meas }}$, the relationship between $W(\alpha)$ and $h_{\text {meas }}$ can be simplified by polynomial regression as presented in Table I. 
Table I

The relationship between $W_{H}$ parameters and $h_{\text {meas }}$ according to a specific attenuation for each magnetic field components.

\begin{tabular}{|c|c|c|c|}
\hline & \multirow{2}{*}{$\begin{array}{l}\text { Attenu } \\
\text { ation }\end{array}$} & \multicolumn{2}{|c|}{ Extraction from electric dipole equation } \\
\hline & & Without Ground plane & With Ground plane \\
\hline \multirow{3}{*}{$W_{H x y}$} & $-3 \mathrm{~dB}$ & $\approx 1.02 \cdot h_{\text {meas }}+0.37 \cdot h_{\text {meas }^{2}}$ & $\approx 0.70 \cdot h_{\text {meas }}-0.1 \cdot h_{\text {meas }}{ }^{2}$ \\
\hline & $-6 \mathrm{~dB}$ & $\approx 1.53 \cdot h_{\text {meas }}+0.64 \cdot h_{\text {meas }}{ }^{2}$ & $\approx 1.01 \cdot h_{\text {meas }}-0.13 \cdot h_{\text {meas }}{ }^{2}$ \\
\hline & $-10 \mathrm{~dB}$ & $\approx 2.16 \cdot h_{\text {meas }}+1.1 \cdot h_{\text {meas }}{ }^{2}$ & $\approx 1.34 \cdot h_{\text {meas }}-0.17 \cdot h_{\text {meas }}{ }^{2}$ \\
\hline \multirow{3}{*}{$W_{H z}$} & $-3 \mathrm{~dB}$ & $\approx 1.39 \cdot h_{\text {meas }}+0.6 \cdot h_{\text {meas }}{ }^{2}$ & $\approx 0.91 \cdot h_{\text {meas }}-0.05 \cdot h_{\text {meas }}{ }^{2}$ \\
\hline & $-6 \mathrm{~dB}$ & $\approx 1.85 \cdot h_{\text {meas }}+1.25 \cdot h_{\text {meas }}{ }^{2}$ & $\approx 1.15 \cdot h_{\text {meas }}-0.025 \cdot h_{\text {meas }^{2}}$ \\
\hline & $-10 \mathrm{~dB}$ & $\approx 2.49 \cdot h_{\text {meas }}+2.85 \cdot h_{\text {meas }^{2}}$ & $\approx 1.42 \cdot h_{\text {meas }}+0.005 \cdot h_{\text {meas }}{ }^{2}$ \\
\hline
\end{tabular}

If $\alpha$ is fixed at $10 \mathrm{~dB}$, the minimum sampling step $W_{H}$ for all the magnetic field components, for the initial dataset, can be estimated by :

$$
W_{H} \leq 2 \cdot h_{\text {meas }}
$$

A progressive spatial sweep is done on a number of sequential step $Q . Q$ could be defined from $d$ and $W_{H}$ according to (17) where $I$ is the biggest integer that validates $W_{H} / d \geq$ $2^{I}$ or according to (18) from the setup parameters definition.

$$
\begin{gathered}
Q=2 \cdot I+1 \\
Q \leq 2 \cdot\left[\log _{2}\left(h_{\text {meas }} / r_{\text {loop }}\right)\right\rfloor+3
\end{gathered}
$$

According to the definition of $Q$, the effective sampling step value $W$, defined by (19) and (20), determines the number of points $N$ collected during the initial step.

$$
\begin{gathered}
W=\mathrm{d} \cdot 2^{(Q-1) / 2} \\
N=\left\lceil\frac{A_{x}}{W / d}-0.5|\cdot| \frac{A_{y}}{W / d}-0.5\right\rceil
\end{gathered}
$$

If the size of $\Omega$ is close to $W$, the number of points collected during the first step could be low. Therefore, it is necessary to add a new constraint on $W$ to ensure a minimum number of points $N$ to start the algorithm. In this case, the problem may also be poorly defined and it can be necessary to increase the size of $\Omega$ to improve $N$.

\section{Definition of selection criterion}

After the collect of the initial dataset, the adaptive part of SSAS algorithm refines the sampling step by step $(q>1)$. The definition of this selection criterion is the main point of this algorithm. The objective is to minimize $M$, by ensuring that the final set of $M$ measurements of $F$ allows building an estimator that minimizes the prediction error of $F$ at any position on $\Omega$. During iteration on the $N q_{\max }$ points in the step $q$, the selection criterion validates whether the value $F\left(p_{q i}\right)$ must be measured in order to minimize $N q$.

This criterion is based on the standard deviation approach calculated from a local estimator $\widehat{F}\left(p_{q i}\right)$ which estimates $F$ at the position $p_{q i}$. Let $P_{M}^{\prime}=\left\{p_{m}\right\}_{m=1}^{\dot{M}} \subset P_{M}$ the temporary dataset resuming all the measurement positions of $F$ already captured. $P_{C}\left(p_{q i}\right)=\left\{p_{c}\right\}_{c=1}^{N_{c}} \subset P_{M}^{\prime}$ defines a local dataset including all the positions neighboring $p_{q i}$ as shown in Fig. 7.
Selection criterion: $\exists p_{c}|| F\left(p_{c}\right)-\hat{F}\left(p_{q i}\right) \mid>k$

In (21), the dataset $F\left(p_{c}\right)$ represents all field values captured at the positions $p_{c}$ of the $P_{C}$ dataset. As resumed by (21), the point $p_{q i}$ is captured whether the field deviation between $\hat{F}\left(p_{q i}\right)$ and $F\left(p_{c}\right)$ is upper than $k . k$ defines the maximum tolerated deviation of the field, between a point and its neighbors in $\mathrm{M}$, to ensure an exact reconstruction of $F$. The definition of $k$ provided in section II.C.2).

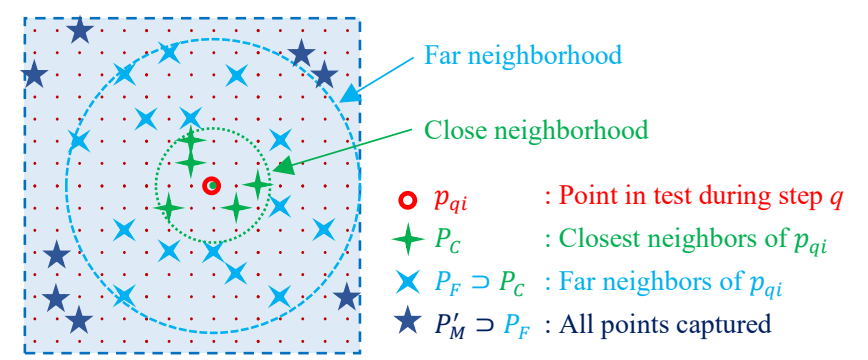

Fig. 7. Definition of the datasets of points in the neighbourhood of $p_{q i}$

\section{1) Definition of the local interpolator}

The selection criterion is computed for each point of $P_{N q} \in$ $P_{M}^{\prime}$. It is mandatory to limit the calculation time of this criterion. The simplest way to create an estimator is to calculate the mean value of the neighbors. To increase the effect of the closest points of $p_{q i}$, the mean value of the neighboring points is weighted by the distance between $p_{q i}$ and each point $p_{c}$. The Inverse Distance Weighting (IDW) interpolator [14] is a widely used method for the spatial estimation (22). Its very short computing time makes it a good choice to compute $\hat{F}\left(p_{q i}\right)$. Note that during the optimization phase, the weighting coefficient value $p$ was fixed at 3 .

$$
\hat{F}\left(p_{q i}\right)=\sum_{j=1}^{N_{c}} \lambda_{j}\left(p_{q i}\right) \cdot F\left(p_{j}\right)
$$

Where:

$$
\lambda_{j}\left(p_{q i}\right)=\frac{w_{j}\left(p_{q i}\right)}{\sum_{l=1}^{N_{c}} w_{l}\left(p_{q i}\right)} ; \quad w_{u}\left(p_{q i}\right)=\left(\frac{1}{d\left(p_{u}, p_{q i}\right)}\right)^{p}
$$

\section{2) Definition of the $k$ parameter}

$k$ defines the acceptable field deviation between two nearest points to keep enough information about $F$. In (21), if $k$ value is low, the probability to capture $p_{q i}$ is high. Otherwise, if $k$ value increases, the probability to capture $p_{q i}$ decreases. In order to adjust the algorithm performance according to the objective, three definitions of $k$ parameter are hereafter proposed. . The section V.B presents a comparative study of the SSAS algorithm using these three $k$ parameters.

According to the definition of $W$ (with $\alpha=10 \mathrm{~dB}$ ), the maximum deviation from the mean was fixed at $10 \mathrm{~dB}$. (24) defines the first simple definition of $k$, used in [9].

$$
k_{1}=10[d B]
$$

Near-field measurement is often used to localize the main root cause of EMC issues, which can be identified by a hot spot of the near-field emission map. From an industrial point of 
view, the maximum level of radiated emission coupled to the accuracy of measurement are the most important information. Therefore, it is mandatory to focus on both parameters during the development of the SSAS algorithm.

The mean error added by the SSAS algorithm degrades the accuracy of measurement. $k_{l}$ must be upgraded to improve the accuracy of measurement around the position of the maximum emission level of $F$. A second definition of $k$ is proposed: $k_{2}$, defined by (25). It takes into account whether the value $\widehat{F}\left(p_{q i}\right)$ is close to the maximum level of $F_{M}^{\prime}$.

$$
k_{2}=K_{A}+\left(K_{B}-K_{A}\right) \cdot \sqrt{\frac{\max \left(F_{M}^{\prime}\right)-\hat{F}\left(p_{q i}\right)}{\max \left(F_{M}^{\prime}\right)-\min \left(F_{M}^{\prime}\right)}}[d B]
$$

Where: $K_{B} \geq K_{A} \geq 0$

Note that $k_{1}$ is a special value of $k_{2}$ where $K_{B}=K_{A}=10 \mathrm{~d} B$.

As shown in Fig. 8, if $\hat{F}\left(p_{q i}\right)$ is close to the maximum level of $F_{M}^{\prime}$ dataset, $k_{2}$ tends to $K_{A}$. Otherwise, if $\hat{F}\left(p_{q i}\right)$ is close tøiel the minimum level of $F_{M}^{\prime}$ dataset, $k_{2}$ tends to $K_{B}$. Both coefficients $K_{A}$ and $K_{B}$ must be defined in order to find a tradeoff between the number of point $M$ and the mesh optimization around the maximum level of $F_{M}^{\prime}$ dataset. According to the tolerated deviation from the mean, $K_{B}$ is set at $k_{1}=10 \mathrm{~dB} . K_{A}$ defines the probability that $p_{q i}$ is captured when $\hat{F}\left(p_{q i}\right)$ is close to the maximum level of $F_{M}^{\prime}$ dataset. If $K_{A}$ is set at zero, all the points around the maximum level will be captured, increasing the number of points $M$. It is accepted that the repeatability of near-field measurement is lower than $1 \mathrm{~dB}$ [1]. So, in order to limit the number of sampling points $M, K_{A}$ is fixed to $1 \mathrm{~dB}$.

The definition of $k_{2}$ is relevant to refine the meshing around the maximum level of the near-field emission map. Note that not all the sources should produce the same maximum level. Actually, several local maxima should likely appear on the near-field emission map. With $k_{2}$ definition, measurement will be only refined around the maximum level of $F$ and not around all these local maxima. To solve this issue, (26) proposes a last improvement of the definition of $k$ parameter.

$$
\begin{aligned}
& k_{3}=K_{A}+\left(K_{B}-K_{A}\right) \cdot \\
& \sqrt{\frac{\max \left(F_{M}^{\prime}\right)-\hat{F}\left(p_{q i}\right)}{\max \left(F_{M}^{\prime}\right)-\min \left(F_{M}^{\prime}\right)} \cdot \min \left(1, \sqrt{\frac{\max \left(F_{F}\left(p_{q i}\right)\right)-\hat{F}\left(p_{q i}\right)}{10}}\right)}[d B]
\end{aligned}
$$

$$
\text { With } \hat{F}\left(p_{q i}\right) \leq \max \left(F_{F}\left(p_{q i}\right)\right) \leq \max \left(F_{M}^{\prime}\right)
$$

$k_{3}$ introduces a proportional coefficient relative to the gap between $\hat{F}\left(p_{q i}\right)$ and $\max \left(F_{F}\left(p_{q i}\right)\right)$ the local maximum value extracted from a local sub-dataset of $F_{M}^{\prime}$ centered around $p_{q i}$ and named $\quad F_{F}\left(p_{q i}\right)=\left\{F\left(P_{F}\left(p_{q i}\right)\right), P_{C} \subset P_{F} \subset P_{M}^{\prime}\right\} . \quad$ Here $P_{F}\left(p_{q i}\right)=\left\{p_{f}\right\}_{f=1}^{N_{F}}$ defines the set of the $N_{F}$ far neighboring positions from $p_{q i}$ as defined in Fig. 7 .

Fig. 8 illustrates the relationship between the different definitions of $k$ parameter and the $\hat{F}\left(p_{q i}\right)$ value. If $\hat{F}\left(p_{q i}\right)$ is close to the $\max \left(F_{M}^{\prime}\right), \max \left(F_{F}\left(p_{q i}\right)\right)$ tends to $\max \left(F_{M}^{\prime}\right)$ resulting that $k_{3}$ tend to $K_{A}$.

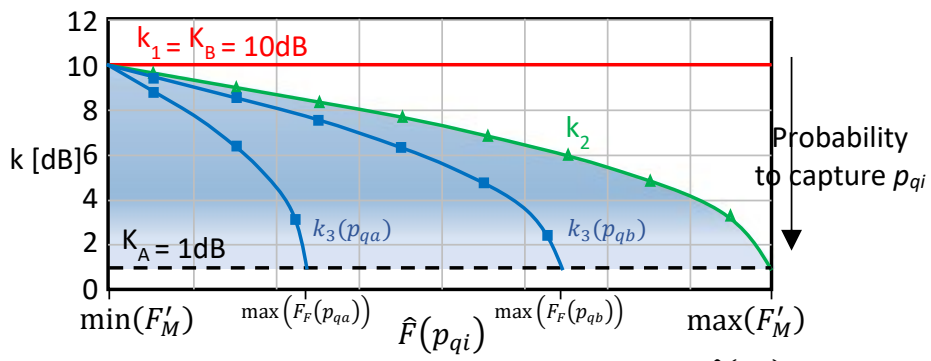

Fig. 8. Relationship between the three $k$ parameters and the $\hat{F}\left(p_{q i}\right)$ value

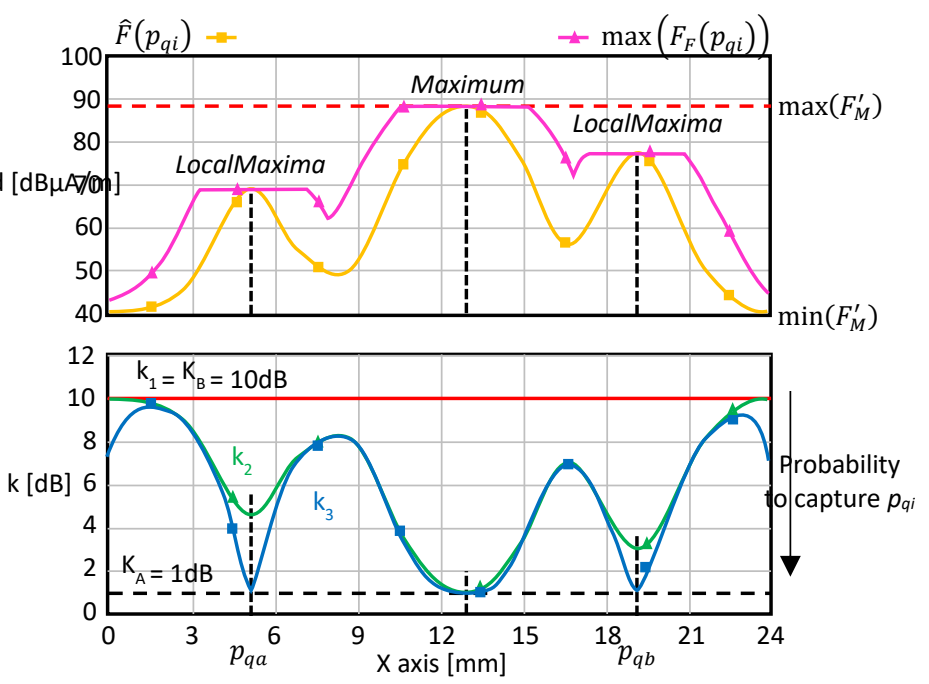

Fig. 9. Evolution of the three $\mathrm{k}$ parameters according to the local field values

Now let's consider $p_{q a}$ close to a local maximum, if $\hat{F}\left(p_{q a}\right)$ tends to $\max \left(F_{F}\left(p_{q a}\right)\right), k_{3}$ also tends to $K_{A}$. Expression of $k_{3}$ builds a tradeoff between the proximity of field value at $p_{q i}$ with both the maximum of $P_{M}^{\prime}$ and the local maxima. $k_{3}$ improves the meshing around all the local maximum level of $F_{M}^{\prime}$ with a meshing enhancement for the highest local maxima. Fig. 9 presents an example of the evolution of the three $k$ parameters values according to the field distribution. For readability purpose, the field is plotted in only one spatial dimension. The difference between $k_{2}$ and $k_{3}$ parameters is clearer in this figure. $k_{3}$ tends to zero for all local maxima $\left(\max \left(F_{M}^{\prime}\right)\right.$ included), leading to a large capture probability, while $k_{2}$ tends to zero only for the $\max \left(F_{M}^{\prime}\right)$. With $k_{3}$, the meshing is more refined around all local maxima.

\section{DEFINITION OF THE INTERPOLATOR}

After SSAS algorithm process, $M$ points irregularly distributed on $\Omega$ are captured. To reconstruct the near-field map with the final resolution $d$, it is necessary to interpolate the field from the $F_{M}$ dataset captured in $\Omega$. The evaluation of the spatial sampling is linked to the performance of the interpolator. The more extensively interpolation is performed the higher is the number of constraints about spatial sampling which must be 
relaxed and hence the number of samples which will be reduced. The most popular spatial interpolation methods are listed below:

- Inverse Distance Weighting (IDW) interpolator [14]

- Triangulated Irregular Network (TIN) interpolator also called Delaunay triangulation interpolator [15]

- $\quad$ Kriging interpolator [16]

\section{A. Kriging interpolator}

The Kriging interpolation method is used here. If we accept a slightly longer computing time, it is the most powerful spatial interpolator among all the methods cited above. The Kriging interpolator, also known as Gaussian Process regression, is an efficient spatial unbiased estimator with minimum variance. The Scikit library on Python [18] has been used to process the adaptive meshing interpolation. The interpolator is initialized with the Exponential kernel.

\section{B. Definition of the comparison indicators}

In order to compare the performance of the SSAS algorithm according to the $W$ and $Q$ parameters (19) and (17), and the choice of the definition of the parameter $k(21)$, it is necessary to define some indicators related to the objective of the SSAS algorithm. The main purpose of this algorithm is to reduce the measurement time, which is directly related to the total number $M$ of measured points. The error at interpolated points is another important indicator. It defines the loss of information resulting of the SSAS algorithm process. The logarithmic error value is computing according to (27).

$$
\operatorname{Error}_{d B}\left(P_{q i}\right)=20 .\left|\log _{10}\left(\hat{F}\left(p_{q i}\right)\right)-\log _{10}\left(F\left(p_{q i}\right)\right)\right|
$$

In (27), $\hat{F}\left(p_{q i}\right)$ represents the interpolated value of $F$ based on Kriging interpolator and $F\left(p_{q i}\right)$ represents the true value of $F$ at the position $p_{q i}$. An important feature of the Kriging interpolator is that it is an unbiased estimator, so that it introduces no interpolation error on the input measurement points $\left(\operatorname{Error}_{d B}\left(F_{M}\right)=0\right)$.

The error is computed only for the highest values of $F$. It is not relevant to compute interpolation error for the field values close to the noise floor of the measurement receiver. So only the points having a field value greater than $\left[\max \left(F_{M}\right)+2 \cdot \min \left(F_{M}\right)\right] / 3$ are used to evaluate the error. The number of points $M$, the mean error and the maximum ErrorontocalMax are the most important indicators to evaluate the performance of the SSAS algorithm.

Note that the maximum error value can be affected by the outlier on the upper tail of the error distribution due to the spatial location error introduced after interpolation process. Therefore, it is not a relevant indicator.

Other indicators can also be added. The $95 \%$ quantile and the number of points affected by an error less than $3 \mathrm{~dB}$ $\left(\mathrm{NbPts}_{\text {error }} \leq 3 \mathrm{~dB}\right)$ are evaluated since they are not affected by the outlier. Error ${ }_{\text {OnMax }}$, defines the error on the maximum of $F$. It is relevant to validate the ability of SSAS algorithm to capture the maximum level of near-field map without significant error. This indicator must be completed by the maximum Errorontocalmax to take into account the error on local maxima of $F$. The complete indicators list used in this study is listed in Table II.

Table II : Indicators for SSAS Algorithm Evaluation

\begin{tabular}{|c|c|c|}
\hline Indicators & Definition & Unit \\
\hline Time & $\begin{array}{l}\text { The complete time of measurement } \\
\text { including } \mathrm{T}_{\text {Algo, }} \mathrm{T}_{\text {Meas }} \text { and } \mathrm{T}_{\text {Move. }}\end{array}$ & [min:s] \\
\hline TAlgo & Algorithm processing time & [min:s] \\
\hline $\mathrm{T}_{\text {Meas }}$ & Time to capture $F_{M}$ & [min:s] \\
\hline TMove & Total time to move probe on $P_{M}$. & [min:s] \\
\hline M & Total number of captured points. & [pts] \\
\hline MeanError & Mean interpolation error value. & {$[\mathrm{dB}]$} \\
\hline ErrorOnLocalMax & $\begin{array}{l}\text { Error values measured on each local } \\
\text { maximum value of } F_{M} \text {. }\end{array}$ & {$[\mathrm{dB}]$} \\
\hline ErrorOnMax & $\begin{array}{l}\text { Error value measured on the maximum } \\
\text { value of } F_{M} \text {. }\end{array}$ & {$[\mathrm{dB}]$} \\
\hline Quantile@95\% & $\begin{array}{l}\text { Maximum value of the } 95 \% \text { of points } \\
\text { with the lowest error. }\end{array}$ & {$[\mathrm{dB}]$} \\
\hline NbPtserror $\leq 3 \mathrm{~dB}$ & $\begin{array}{l}\text { Number of points where the interpolation } \\
\text { error is lower than } 3 \mathrm{~dB} \text {. }\end{array}$ & {$[\%]$} \\
\hline
\end{tabular}

\section{Presentation of the Validation Case Studies}

\section{A. Case study \#1 from simulation data: "Dipoles"}

The first proposed case study (Fig. 10) is a $75 \mathrm{~mm} \times 75 \mathrm{~mm}$ printed circuit board (PCB) simulation model including six traces routed in air at $1 \mathrm{~mm}$ above an ideal ground plane.
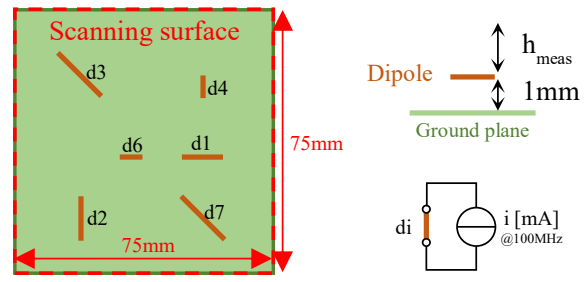

\begin{tabular}{|c|c|}
\hline Dipoles & $\begin{array}{c}\text { Current } \\
{[\mathrm{mA}]}\end{array}$ \\
\hline $\mathrm{d} 1$ & 0.03 \\
$\mathrm{~d} 2$ & 0.01 \\
$\mathrm{~d} 3$ & 0.33 \\
$\mathrm{~d} 4$ & 1 \\
$\mathrm{~d} 5$ & 0.001 \\
$\mathrm{~d} 6$ & 1 \\
\hline
\end{tabular}

Fig. 10. Description of case study \#1 "Dipoles"

The six traces have the same width equal to $0.05 \mathrm{~mm}$ and are routed in three different directions $\left(0^{\circ}, 45^{\circ}\right.$ and $\left.90^{\circ}\right)$. They are excited by a sinusoidal current source at $100 \mathrm{MHz}$. The magnitude of the three magnetic field components radiated by the wire is computed [19] from analytical equations (4)(5) at several heights $h_{\text {meas }}(1,5$ and $18 \mathrm{~mm})$. Image theory is used to take into account the ideal ground plane. The sampling resolution $d$ is $0.5 \mathrm{~mm}$.

\section{B. Case study \#2 from measurement data: "FPGA"}

Fig. 14 presents the XILINX Spartan 6 FPGA device test board. The frequency range of the magnetic near-field measurements starts at $5 \mathrm{MHz}$ and increases up to $1.8 \mathrm{GHz}$. Two types of magnetic field probe are used: one for the $\mathrm{Hx}$ and $\mathrm{Hy}$ components and another for the $\mathrm{Hz}$ component. The spatial resolution of the probes is $0.5 \mathrm{~mm}$. The probes are moved at 1.1 $\mathrm{mm}$ above the FPGA package. The height of measurement is fixed at $h_{\text {meas }}=1.85 \mathrm{~mm}$ above the top of the IC substrate. More information about the configuration of the FPGA is presented in [17].

For both case studies, it is possible to define the theoretical optimal configuration of $W$ and $Q$ parameters according to (19) and (18), as summarized in Table III. 


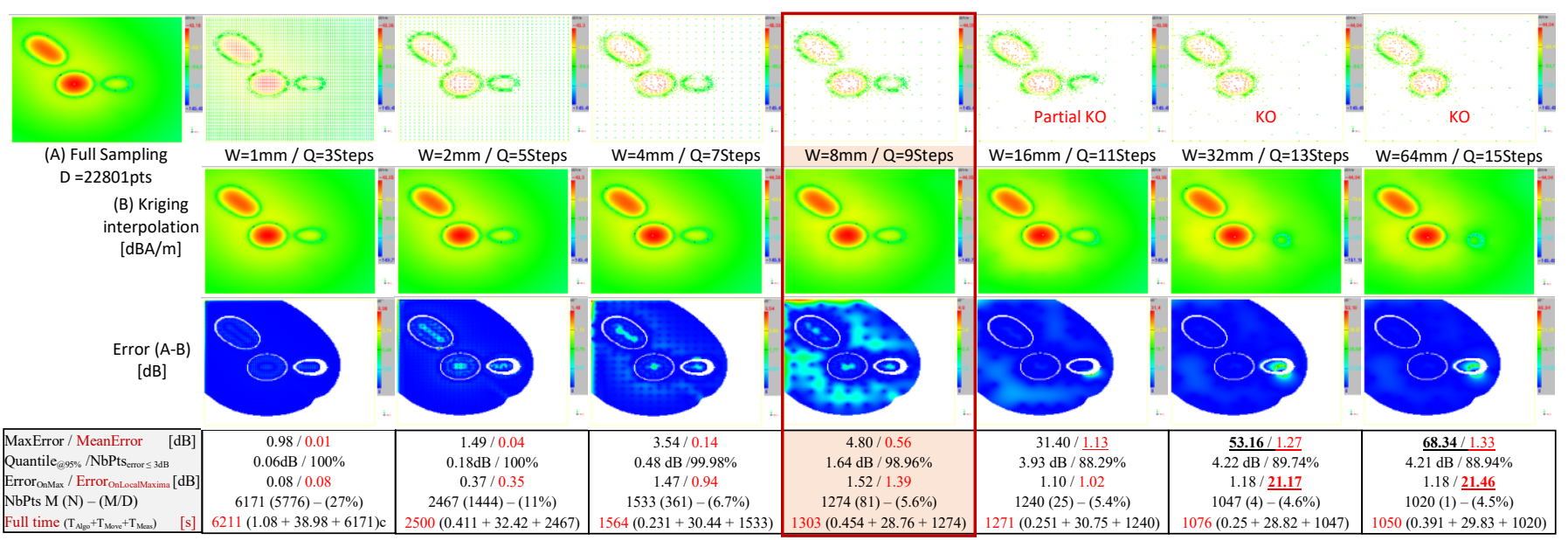

Fig. 11. Evaluation of the impact of $W$ and $Q$ parameters : Case Study \#1 "Dipoles" (Hy measurement, $h_{\text {meas }}=5$ mm, Selection criterion= $k_{l}$ ) (Note: "KO" means that the reconstructed of the near-field map is erroneous)

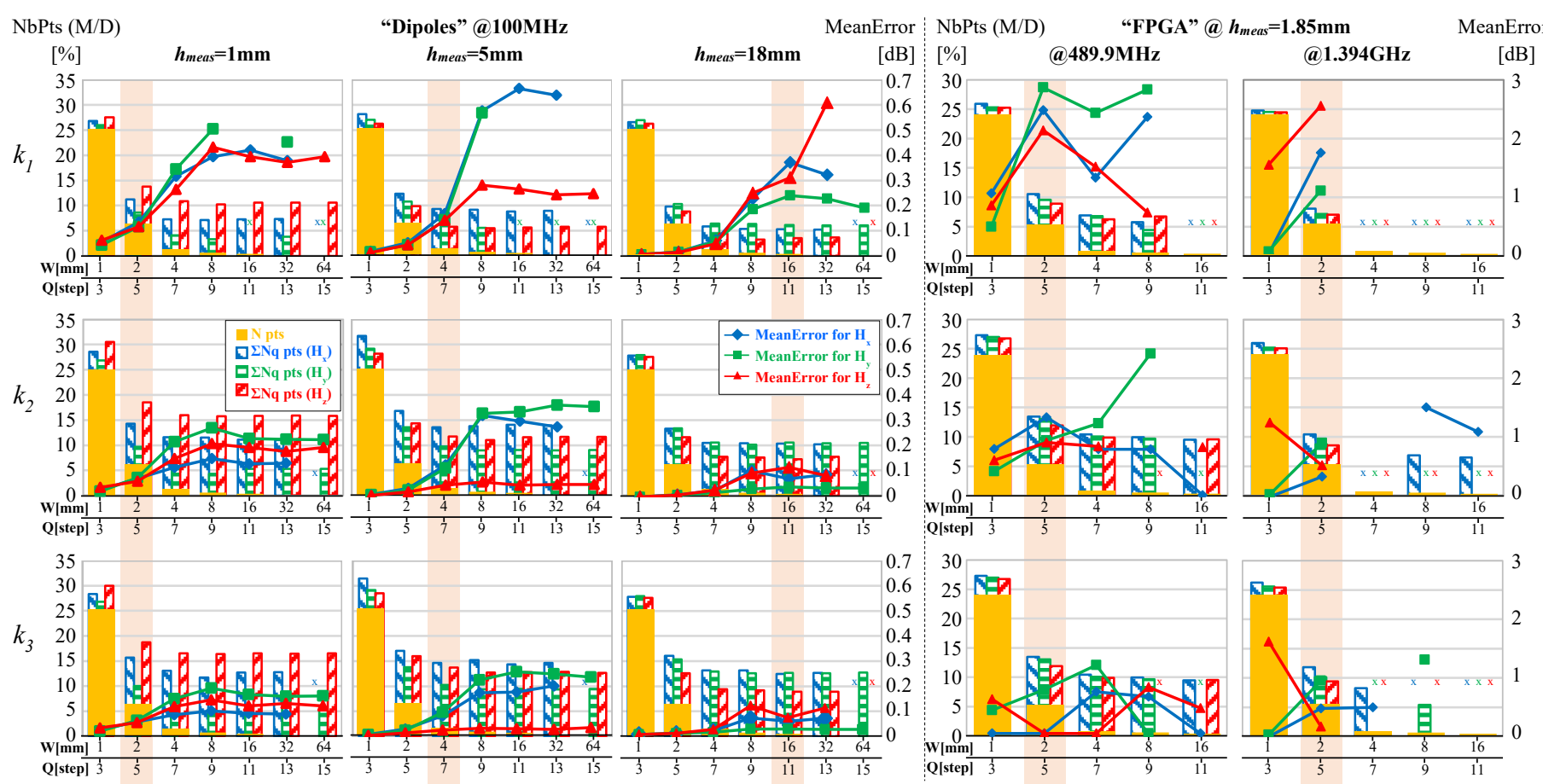

Fig. 12. Evaluation of the impact of $\mathrm{W}, \mathrm{Q}$ and $\mathrm{k}$ parameters on both case study (Note: " $x$ " means the reconstructed of the near-field map is erroneous)

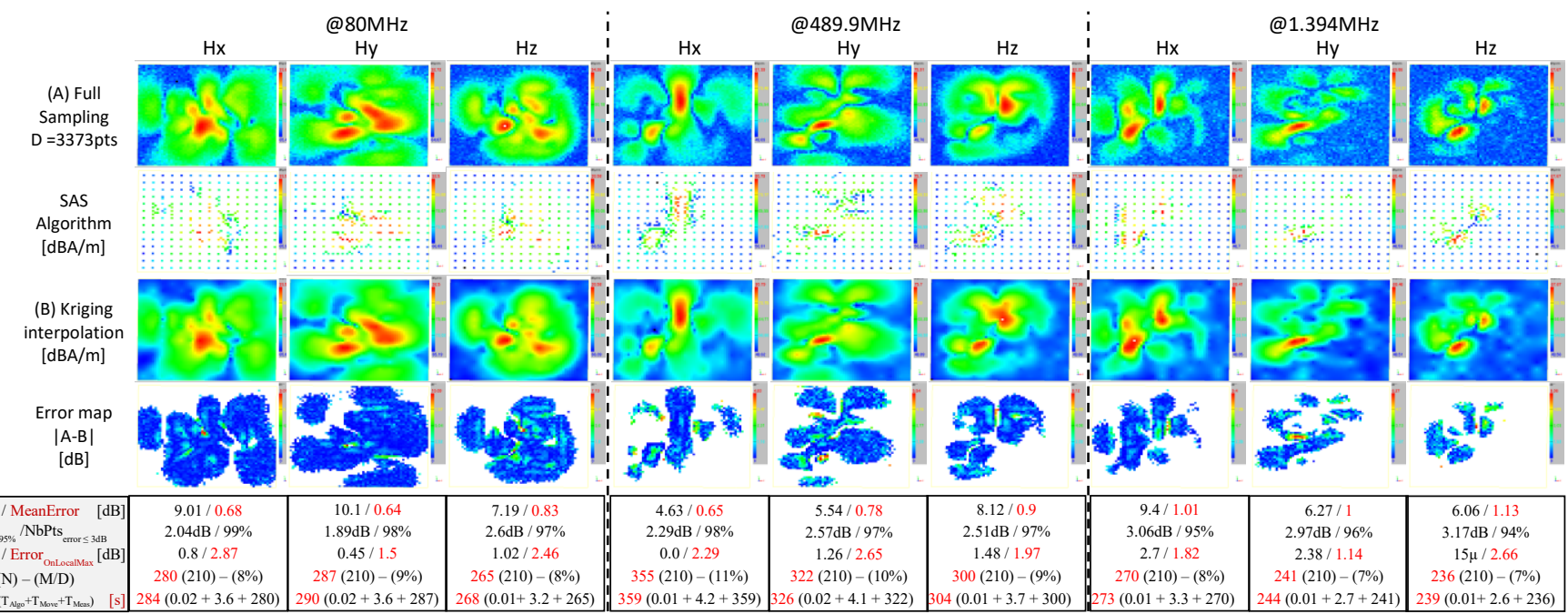

Fig. 13. Near field measurement results vs. frequency for each field component on the case study \#2 "FPGA" $-\mathrm{k} 1-5$ Steps 


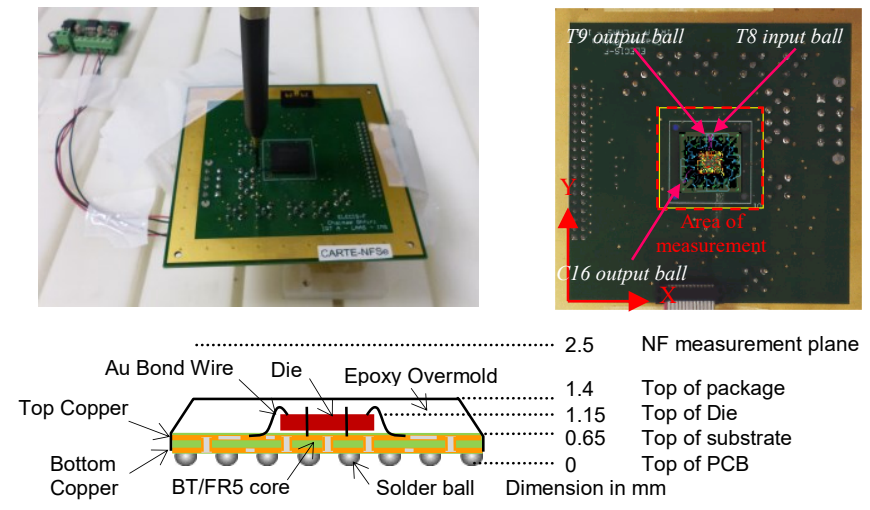

Fig. 14. Description of cases studies \#2 : "FPGA"

Table III : SSAS Algorithm Parameters

\begin{tabular}{|c|c|c|c|}
\hline \multirow{2}{*}{ Parameters } & \multicolumn{2}{|c|}{ Values for case studies } & \multirow{2}{*}{ Unit } \\
\hline & \#1 & \#2 & \\
\hline & 0.5 & 0.5 & [mm] \\
\hline & $1 / 5 / 18$ & 1.85 & [mm] \\
\hline$Q \leq 3+2 \cdot\left\lfloor\log _{2}\left(h_{\text {meas }} / r_{\text {loop }}\right)\right\rfloor$ & $5 / 9 / 13$ & 5 & [step] \\
\hline$W=r_{\text {loop }} \cdot 2^{(Q-1) / 2}$ & $2 / 8 / 32$ & 2 & [mm] \\
\hline
\end{tabular}

Data of both case studies, including measurements, are available [20] for non-commercial and academic use only.

\section{VALidATION OF THE PROPOSEd SSAS Algorithm}

\section{A. Discussion about $W$ and $Q$ parameters}

The parameters $d, W$ (and $Q$ ) initialize the SSAS algorithm. $W$ and $Q$ are coupled and define the resolution of the first step where all points are captured. Expression (19), in section II, gives the optimal expression of $W$ according to the measurement height $h_{\text {meas }}$. Fig. 11 and Fig. 12 present the results of the SSAS algorithm applied on case study \#1 for several measurement heights and several values of the parameter $W$ (and $Q$ ).

The configuration for the optimal value of $W$ (and $Q$ ) is underlined in light red in Fig. 11 and Fig. 12. It can be observed that the optimal values of $W$ and $Q$ offer the best configuration to minimize the number of points while maintaining the lowest mean error. If $W$ is set with a greater value than this optimal value proposed in (19), the number of measurement points is not significantly reduced but the interpolation error increases rapidly leading to an erroneous reconstruction of the near-field map. This situation is marked in Fig. 12 by ' $x$ ' including the number of points at zero and no value for the mean error. It is particularly true when the $k_{1}$ criterion is used. It seems that criteria $k_{2}$ and $k_{3}$ are more robust than $k_{1}$. These results validate the optimal choice of $W$ and $Q$ which are defined by the simple equations (19) and (18) according to the measurement configuration ( $h_{\text {meas }}$ and $\left.r_{\text {loop }}\right)$.

\section{B. Discussion about selection criteria}

Three selection criteria were introduced in section II.C. The selection criterion must be chosen according to the measurement objective. The selection criterion $k_{l}$ is the best choice to reduce the measurement time at the price of an increase in the interpolation error. $k_{2}$ criterion reduces the interpolation error around the point of maximum level and $k_{3}$ around all the local maxima. However, it leads to an increase of measurement points. This general conclusion is illustrated in Fig. 15 which shows an evaluation of the number of points $M$, the MeanError and the maximum ErroronLocalMax for both case studies using the three selection criteria.

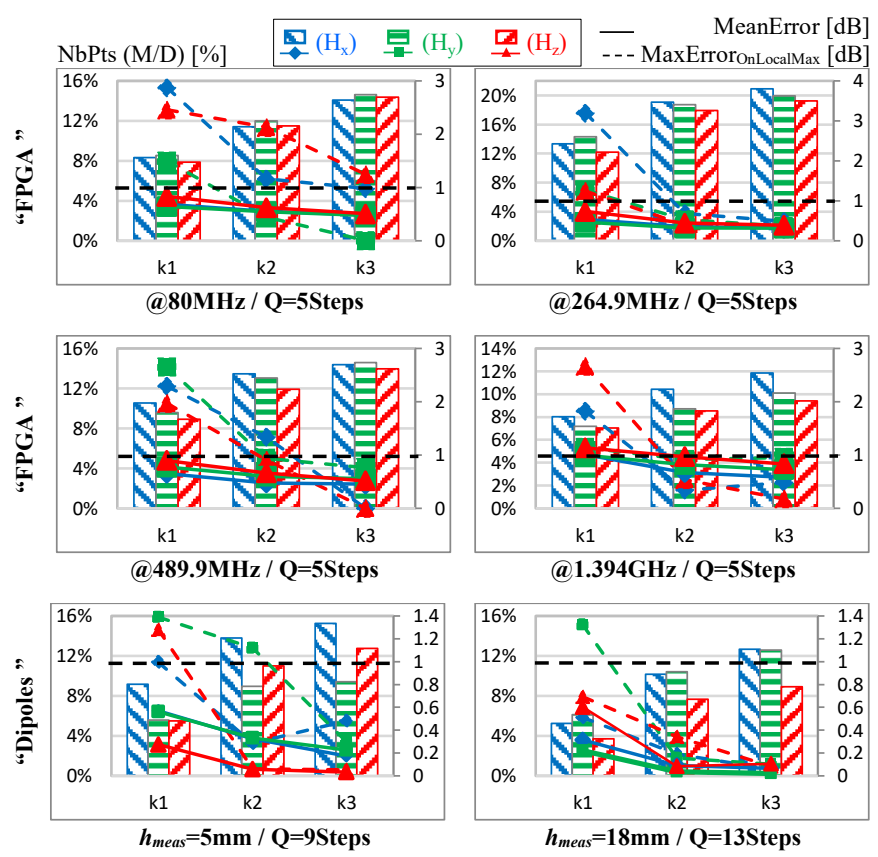

Fig. 15. Comparison of the SSAS algorithm performances according to the three selection criteria for both case studies

Fig. 16 completes this evaluation in a large frequency range. Using $k_{3}$ criterion, the error value can be greatly reduced (the MeanError $<1 \mathrm{~dB}$ and the MaxErroronLocalMax $<2 \mathrm{~dB}$ ) without a significant increasing of the number of points. Compared to the $k_{1}$ and $k_{2}$ criteria, using $k_{3}$ criterion leads to an increase of 3 to $6 \%$ in the measurement points, which never exceeds $21 \%$ of the total number of points of the near-field map with the final resolution $d$. With $k_{1}$, the number of measurement points never exceeds $15 \%$ of the total number of points, the MeanError value remains less than $1.6 \mathrm{~dB}$, but the MaxErroronLocalMax value can reach up to $8 \mathrm{~dB}$.

Fig. 17 presents the spatial meshing characteristics according to the three selection criteria. Using $k_{l}$ reduces significantly the number of points to refine the meshing around area with high field deviation. However, due to the low field deviation around the strongest values, the actual maximum is likely going to be missed out. Using $k_{2}$ improves this issue by refining the meshing around the position of the maximum of field without affecting the local maxima. With $k_{3}$, the sampling around the maximum of field and all maxima is improved, leading to a significant reduction of the interpolation error as confirmed by Fig. 15.

As presented by Fig. 13 and Fig. 15, the frequency does not affect the performance of the SSAS algorithm. Only the spatial distribution of the field determines the reduction of the number of points. The larger the dispersion of the near-field values spatial distribution, the higher is the number of points increased 
by the SSAS algorithm in order to keep enough information on $F$ and hence to ensure a satisfactory reconstruction of the field within $\Omega$.

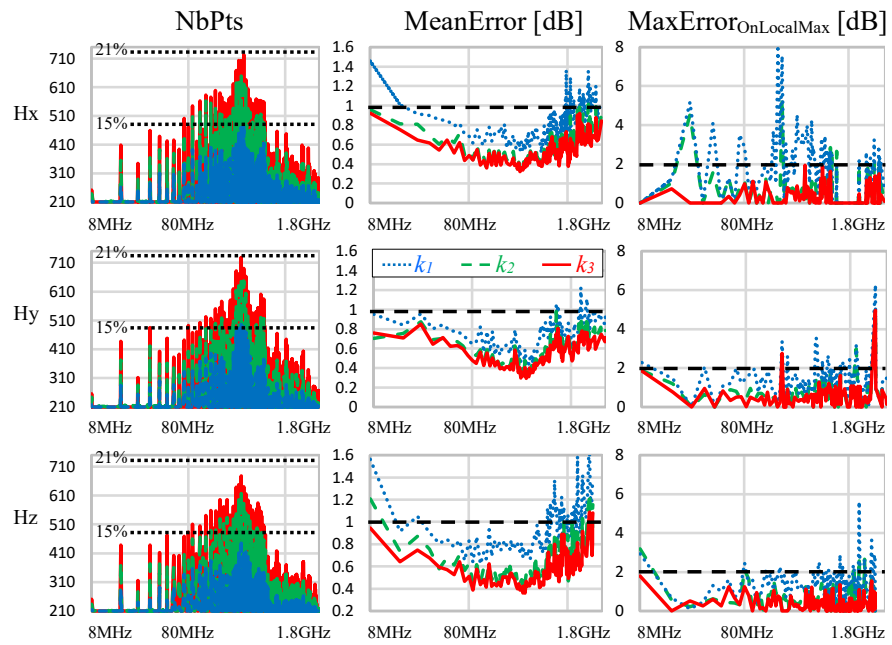

Fig. 16. The evaluation of the errors indicators vs. frequency according to the three selection criteria on FPGA case study $(\mathrm{Q}=5$-Steps $)$

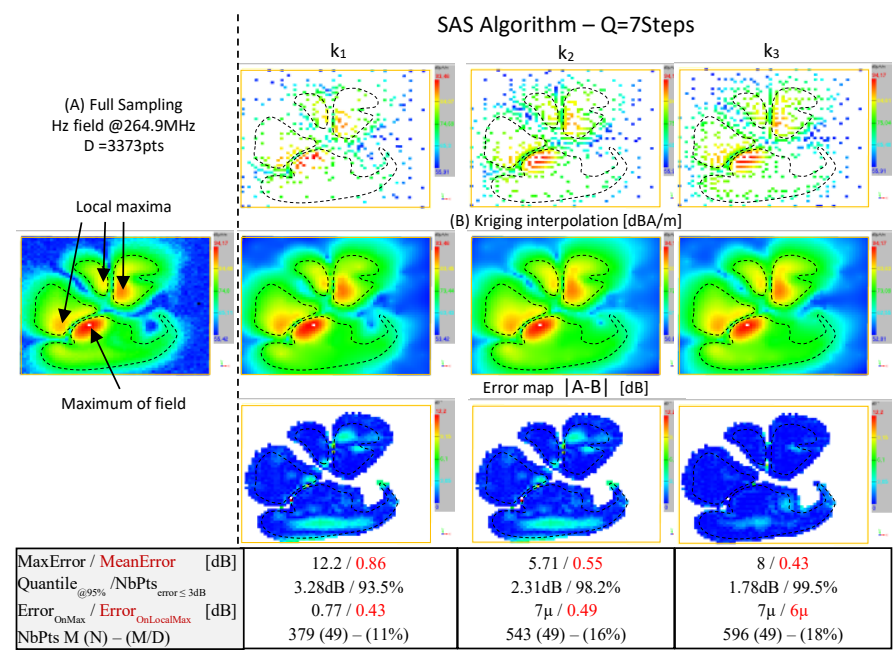

Fig. 17. Spatial meshing evaluation according to the three selection criteria on FPGA case study-Hz field component @264.9MHz

\section{Discussion about the measurement time optimization}

The measurement time optimization is the main objective of this study. Fig. 18 shows a comparison of the measurement time according to the selection criterion. The duration of the acquisition of $F_{M}$ by measurement equipment ( $\mathrm{T}_{\text {Meas }}$ ), the nearfield probe displacement time on $P_{M}\left(\mathrm{~T}_{\text {Move }}\right)$ and the execution time of the SSAS algorithm $\left(\mathrm{T}_{\mathrm{Algo}}\right)$ are analyzed separately. Noteworthy is that the trends of these three durations are not impacted by the frequency. They only depend from the number of points. In order to calculate the measurement time, one second per point is taken as assumption. A constant speed of 20 $\mathrm{cm} / \mathrm{s}$ is considered to compute the probe displacement time (probe acceleration and deceleration phases are not taken into account).

The SSAS algorithm reduces the number of measurement points in order to select only the points that give the most information about $F$. As shown in Fig. 18, the acquisition time, which is proportional to the number of measurement points, occurs for $99 \%$ of the measurement time ( $T_{c t r l}$ is here neglected). Whatever is the configuration, the probe displacement time and especially the SSAS algorithm processing time are negligible.

SSAS algorithm processing represents less than $0.1 \%$ of total measurement time. It validates our objective to develop a low time-consuming algorithm. The probe displacement time is negligible compared to the acquisition time since it represents less than $1 \%$ of the total measurement time. This is the benefit of using a regular mesh (Sukharev grid) and a progressive sequential sweep described in Fig. 2. Moreover, the distance between two consecutive points is minimized.

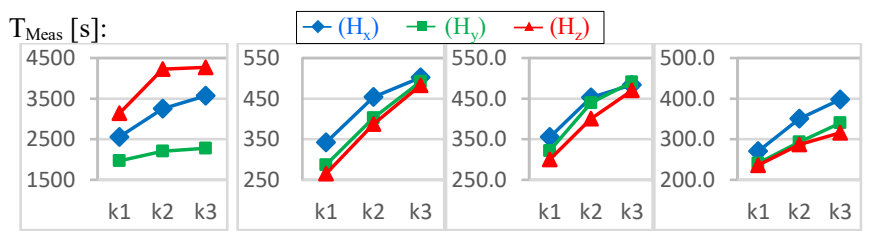

$\mathrm{T}_{\text {Move }}[\mathrm{s}]$ :

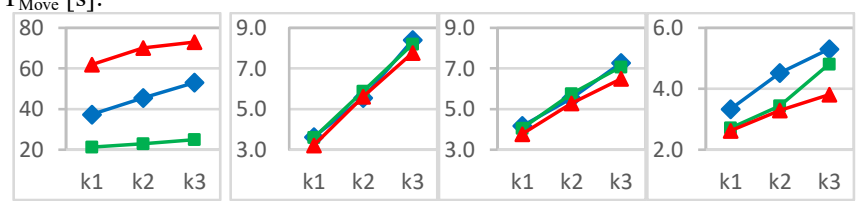

$\mathrm{T}_{\text {Algo }}[\mathrm{s}]$ :

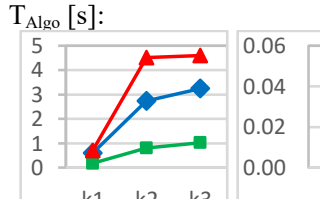

\begin{tabular}{lll|l} 
k1 & $k 2$ & k3
\end{tabular}

Dipoles@100MHz $\boldsymbol{h}_{\text {meas }}=5 \mathrm{~mm} / \mathrm{Q}=5$ Steps

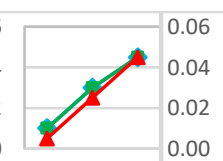

k1 $\quad$ k2 $\quad$ k3

(a) $80 \mathrm{MHz}$

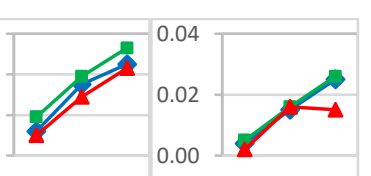

$\begin{array}{llllll}\text { k1 } & \text { k2 } & \text { k3 } & \text { k1 } & \text { k2 } & \text { k3 }\end{array}$

FPGA - $h_{\text {meas }}=1.85 \mathrm{~mm} / \mathrm{Q}=5$ Steps
Fig. 18. Comparison of the different contributions to the measurement duration according to the selection criterion of the SSAS algorithm

Table IV summarizes the measurement times with the full grid measurement and using SSAS algorithm configured with the three selection criteria. On case study \#1 "Dipoles", SSAS algorithm reduces measurement time to half an hour whereas the full measurement lasts more than six hours. SSAS algorithm ensures a reduction of the measurement time according to the frequency by a factor of 7 to 13 using selection criterion $k_{l}$ and by a factor of 5 to 10 using $k_{3}$.

Table IV

COMPARISON OF THE MEASUREMENT TIMES WITH THE DIFFERENT SELECTION CRITERION ON BOTH CASE STUDIES

\begin{tabular}{|c|c|c|c|c|}
\hline \multirow[t]{2}{*}{ Configuration (Hy) } & \multirow{2}{*}{$\begin{array}{c}\text { Full } \\
\text { Sampling }\end{array}$} & \multicolumn{3}{|c|}{ Selection criterion } \\
\hline & & $\mathrm{k}_{1}$ & $\mathrm{k}_{2}$ & $\mathrm{k}_{3}$ \\
\hline \multicolumn{5}{|l|}{ "Dipoles" } \\
\hline$h_{\text {meas }}=1 \mathrm{~mm} ; \mathrm{Q}=5$ steps & $6 \mathrm{~h} 20 \mathrm{~m} 58 \mathrm{~s}$ & $33 \mathrm{~m} 08 \mathrm{~s}(9 \%)$ & $37 \mathrm{~m} 04 \mathrm{~s}(10 \%)$ & $38 \mathrm{~m} 23 \mathrm{~s}(10 \%)$ \\
\hline$h_{\text {meas }}=5 \mathrm{~mm} ; \mathrm{Q}=9$ steps & $6 \mathrm{~h} 20 \mathrm{~m} 58 \mathrm{~s}$ & $21 \mathrm{~m} 43 \mathrm{~s}(6 \%)$ & $34 \mathrm{~m} 42 \mathrm{~s}(9 \%)$ & $36 \mathrm{~m} 31 \mathrm{~s}(10 \%)$ \\
\hline$h_{\text {meas }}=18 \mathrm{~mm} ; \mathrm{Q}=13$ steps & $6 \mathrm{~h} 20 \mathrm{~m} 58 \mathrm{~s}$ & $24 \mathrm{~m} 12 \mathrm{~s}(6 \%)$ & $40 \mathrm{~m} 59 \mathrm{~s}(11 \%)$ & $49 \mathrm{~m} 27 \mathrm{~s}(13 \%)$ \\
\hline \multicolumn{5}{|l|}{ "FPGA" } \\
\hline (a) $80 \mathrm{MHz} ; \mathrm{Q}=5$ steps & $56 \mathrm{ml} 1 \mathrm{~s}$ & $4 \mathrm{~m} 50 \mathrm{~s}(9 \%)$ & $6 \mathrm{~m} 48 \mathrm{~s}(12 \%)$ & $8 \mathrm{~m} 20 \mathrm{~s}(15 \%)$ \\
\hline (a) 264.9MHz; Q=5steps & $56 \mathrm{ml} 1 \mathrm{~s}$ & $8 \mathrm{~m} 08 \mathrm{~s}(14 \%)$ & $10 \mathrm{~m} 39 \mathrm{~s}(19 \%)$ & $11 \mathrm{~m} 22 \mathrm{~s}(20 \%)$ \\
\hline (a) $489.9 \mathrm{MHz} ; \mathrm{Q}=5$ steps & $56 \mathrm{ml} 1 \mathrm{~s}$ & $5 \mathrm{~m} 26 \mathrm{~s}(10 \%)$ & $7 \mathrm{~m} 24 \mathrm{~s}(13 \%)$ & $8 \mathrm{~m} 18 \mathrm{~s}(15 \%)$ \\
\hline (a) $1.394 \mathrm{GHz} ; \mathrm{Q}=5 \mathrm{steps}$ & $56 \mathrm{ml1s}$ & $4 \mathrm{~m} 03 \mathrm{~s}(7 \%)$ & $4 \mathrm{~m} 55 \mathrm{~s}(9 \%)$ & $5 \mathrm{~m} 44 \mathrm{~s}(10 \%)$ \\
\hline
\end{tabular}

\section{CONCLUSION}

The main objective of this work was to reduce the 
measurement time of the near-field scanning of electronic equipment. To reach this goal, an existing progressive Sequential Spatial Adaptive Algorithm [9] has been updated and improved. The proposed SSAS algorithm presents a negligible computation time. In fact, depending from the device under test, the measurement time is reduced by a factor 5 to 13 according to the spatial distribution of $F$ and the choice of the selected criterion.

Two points ensure the accuracy of the measurement method. At first, the $W$ parameter definition guarantees that the minimal dataset is collected during the first acquisition step. Next, this paper presents two new selection criteria to complete the first proposal method [9]. A special attention has been given in the selection criterion definition to limit the error around the local maxima of the near-field map. The proposed approach keeps this error lower than $1 \mathrm{~dB}$, which is the estimated near-field measurement error.

The proposed selection criteria are based only on the magnitude of the field captured at one frequency. The performances of this algorithm on multi-frequency range and vector measurements have not been evaluated. The definition of a specific criterion would be certainly necessary. These questions will be addressed in future works.

\section{REFERENCES}

[1] S. Serpaud and S. Leman, "Technical improvement of the near field measurement: The vector near field scanning," in Proc. EMC Eur., Sep. 2-6, 2013, pp. 711-716.

[2] M. Rahimi, M. Hansen, W. J. Kaiser, G. S. Sukhatme and D. Estrin, "Adaptive sampling for environmental field estimation using robotic sensors," 2005 IEEE/RSJ International Conference on Intelligent Robots and Systems, Edmonton, Alta., 2005, pp. 3692-3698, doi: 10.1109/IROS.2005.1545070.

[3] T. Hachisuka, W. Jarosz, R. P. Weistroffer, K. Dale, G. Humphreys, M. Zwicker, H. W. Jensen. "Multidimensional adaptive sampling and reconstruction for ray tracing," ACM Trans. Graph. 27, 3 (August 2008), 1-10. DOI:https://doi.org/10.1145/1360612.1360632

[4] M. A. Qureshi, C. H. Schmidt, T. F. Eibert, "Adaptive Sampling in Spherical and Cylindrical Near-Field Antenna Measurements," IEEE Antennas and Propagation Magazine 55(1): pp. 243-249, February 2013, DOI. 10.1109/MAP.2013.6474537.

[5] R. Xu,1 X. Zhou, Y. Hirano, R. Tachibana, T. Hara, S. Kido, H. Fujita, "Particle System Based Adaptive Sampling on Spherical Parameter Space to Improve the MDL Method for Construction of Statistical Shape Models," Computational and Mathematical Methods in Medicine, Vol. 2013, pp 9, http://dx.doi.org/10.1155/2013/196259

[6] R. Theunissen1 and P. Gjelstrup, "Adaptive sampling in two dimensions for point-wise experimental measurement techniques," in Proc. 19th Int. Symp. Appl. Laser Imag. Techn. Fluid Mech., Jul. 16-19, 2018, pp. 10351048 .

[7] D. Deschrijver, D. Pissoort, T. Dhaene, "Automated near-field scanning algorithm for the EMC analysis of electronic devices," IEEE Transactions on EMC, vol. 54, no. 3, pp. 502-510, June 2012.

[8] T. Dorné et al., "Optimized sequential sampling algorithm for EMI nearfield scanning," in Proc. EMC Eur., Brugge, Sep. 2-6, 2013, pp. 385-388.

[9] S. Serpaud, A. Boyer, and S. Ben Dhia, "Sequential adaptive sampling algorithm to reduce the near-field measurement time," in Proc. EMC-Eur., Sep. 2-6, 2019, pp. 680-685, doi: 10.1109/EMCEurope.2019.8872034.

[10] IEC 61967-3, TS, Ed.1: Integrated circuits- Measurement of electromagnetic emissions, $150 \mathrm{KHz}$ to $1 \mathrm{GHz}$ - Part 3: Measurement of radiated emissions - Surface scan method.

[11] A. Brandt. "Multi-level adaptive solutions to boundary-value problems," Mathematics of Computation, vol. 31, pp 333-390, 1977.
[12] S. M. LaValle, M. S. Branicky, S. R. Lindemann, "On the Relationship between Classical Grid Search and Probabilistic Roadmaps," The International Journal of Robotics Research, 23(7-8), pp. 673-692, 2004, DOI. 10.1177/0278364904045481.

[13] I. V. Lindell, E. Alanen, "Exact image theory for the Sommerfeld halfspace problem, Part I: Vertical magnetic dipole," IEEE Trans. An-tennas Propagat., vol. AP-32, pp. 126-133, Feb. 1984.

[14] D.Shepard, "A two-dimensional interpolation function for irregularlyspaced data," Proceedings of the 1968 ACM National Conference, pp. 517-524, DOI. 10.1145/800186.810616.

[15] B. Delaunay "Sur la sphère vide, A la mémoire de Georges Voronoï," bulletin de l'académie des sciences de l'URSS, 6, 793-800, 1934.

[16] D. G. Kriege, "Two dimensional weighted average trend surfaces for ore evaluation," inProc. J. South African Inst. Mining Metall., Proc. Symp. Math. Statist. Comput., Mar. 1966, pp. 7-8.

[17] S. Serpaud, C. Ghfiri, A. Boyer and A. Durier, "Proposal for combined conducted and radiated emission modelling for Integrated Circuit," 2017 11th International Workshop on the Electromagnetic Compatibility of Integrated Circuits (EMCCompo), St. Petersburg, 2017, pp. 172-177, doi: 10.1109/EMCCompo.2017.7998105.

[18] The Python Scikit library v0.21.3-2019.[Online] - https://scikit-learn.org

[19] IC-EMC 2v9 freeware - 2020.[Online] - https://www.ic-emc.org

[20] Case studies data - 2020.[Online] - http://ic-emc.org/nefisc-editor.html

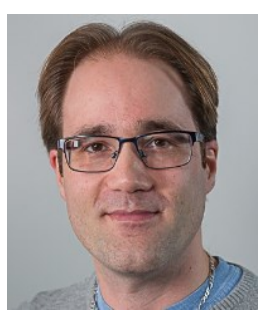

Sébastien Serpaud (Student Member, IEEE) received the engineering professional qualification in Electronic and Computer Science and the Master's degree in Microelectronic and Microsystems circuit design in 2006 from the National Institute of Applied Sciences (INSA) of Toulouse, France.

In 2006, he joined Freescale Semiconductor in research activity about EMC for semiconductors manufacturing. From 2007 to 2017, he was an EMC research engineer with Nexio. In 2015, he joined IRT SaintExupéry institute, Toulouse, France, where he is currently a team leader of the research group on EMC field. Since 2018, he has been working toward the Ph.D. degree at INSA, Toulouse, France, in application of the near-field emission measurement method to improve the design and the investigation of EMC. His research interests include development of investigation tools around the near-field measurement, the EMC modeling at IC and electronic board level, and freeware tools development for EMC (NeFiSc Editor and Touchstone Editor) http://emctools.free.fr.

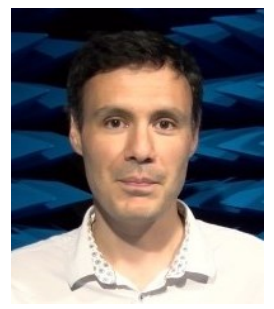

Alexandre Boyer obtained a Masters degree in electrical engineering in 2004 and a PhD in Electronics from the Institut Nationale des Sciences Appliquées (INSA) in Toulouse, France, in 2007. He is currently an Associate Professor in the Department of Electrical and Computer Engineering at INSA, Toulouse. He is leading his research at the Laboratoire d'Analyse et d'Architecture des Systèmes (LAAS-CNRS), as part of the 'Energy and Embedded Systems' research group. His current research interests include EMC measurements, IC EMC and reliability modeling, and computer aided design (CAD) tool development for EMC (IC-EMC freeware).

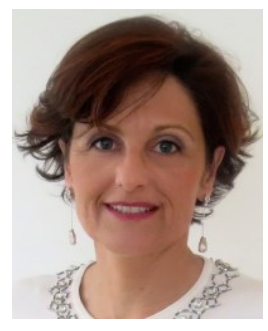

Sonia Ben Dhia obtained her Masters degree in electrical engineering in 1995, and a Ph.D. in Electronic Design from the Institut National des Sciences Appliquées (INSA), Toulouse, France, in 1998. Full professor at INSA-Toulouse (French engineering institute), Department of Electrical and Computer Engineering, she teaches digital electronics, IC testability and reliability, and analog and RF CMOS design. CEO of INSA Euro-Méditerranée, Fès, Morocco (2014-2017), 
she was responsible for the overall leadership and management of this new engineering institute. Her research interests at LAAS - CNRS Toulouse include signal integrity in nano-scale CMOS ICs, electromagnetic compatibility and reliability of ICs. She has authored and co-authored 3 books, more than 120 publications in peer-reviewed journals \& conference proceedings and supervised $14 \mathrm{PhD}$ theses and 9 M.Sc. theses.

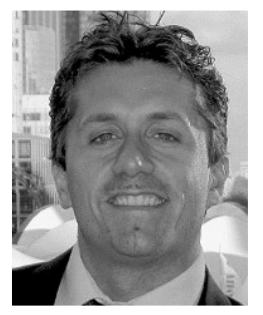

Fabio Coccetti received the Laurea M.S. degree in electrical engineering from the University of Perugia, Perugia, Italy, the Ph.D. degree in high frequency engineering at the Technische Universitt Mnchen (TUM) in Munich Germany, and the Habilitation direction de recherche (HDR) from INP-Toulouse, in 1999, 2004, and 2013, respectively.

$\mathrm{He}$ has been visiting scientist at University of Michigan USA in 2000 and from 2004 to 2008, he has been working as research scientist at the Laboratoire dAnalyse et dArchitectures des Systmes at the Centre National de la Recherche Scientifique (LAAS-CNRS) inToulouse, France. From 2008 to 2016 he has been project manager at Elemca (Toulouse) and from 2016 to 2018 general manager of RF Microtech France. Since March he joined the IRT Saint Exupery as head of the Component Modeling and Reliabilty competence center within the the More Electrical Aircraft Department. Dr. Coccetti has been involved in numerous research projects going from the theoretical and experimental investigation of power handling in RF-MEMS to design modelling and experimental validation of reliability for Microelectronics, nanosystems and more recently power electronics. Dr. Coccetti has more than 150 publications in peer reviewed conference and journal papers, and he is co-author $\mathrm{s}$ of two book chapters. He is has been European Microwave Lecturer (2013-2016) and member of the European Microwave Association (EUMA). He is member of the IEEE society, cofounder and chair of the RFNanotechnology Committee within the IEEE-MTT society. He was the recipient of the IHP InternationalWolfgang Mehr Fellowship Award in 2016. 